\title{
Study of Magnetic Inhomogeneity on the Magnetoimpedance Effect of Soft Magnetic Alloys
}

\author{
S.M. MohsenI ${ }^{a *}$ AND M.M. TEhranChI ${ }^{a, b}$ \\ ${ }^{a}$ Laser and Plasma Research Institute, G.C. \\ Shahid Beheshti University, Evin, Tehran 1983963113, Iran \\ ${ }^{b}$ Physics Department, G.C., Shahid Beheshti University \\ Evin, Tehran 1983963113, Iran
}

\begin{abstract}
There are various annealing techniques to influence the magnetic anisotropy of amorphous soft magnetic alloys, applicable for magnetic sensing elements. Laser irradiation of amorphous magnetic ribbons is a kind of annealing method to affect their magnetic moment orientations and consequently change their magnetic anisotropy and magnetoimpedance effect. In this paper, we have studied the role of magnetic anisotropy in the magnetoimpedance effect of laser annealed amorphous ribbons. In our theoretical study, we took into account the variations of magnetic anisotropy constants of local magnetic moments after annealing process. We showed that the flatten magnetoimpedance response which was observed in the experiments can be justified by averaging different local anisotropies induced by laser processing.
\end{abstract}

PACS numbers: 75.30.Gw, 75.50.Kj, 75.60.-d, 75.40.Mg

\section{Introduction}

Developing magnetic materials capable of applications in magnetic sensing elements requires an understanding of the fundamental physics behind their experimental phenomena. Soft magnetic amorphous alloys are among the interesting materials for study and application, owing to their unique magnetic properties such as magnetic anisotropy, magnetotransport, and magnetization dynamics [1]. Magnetoimpedance (MI) effect which is the electrical impedance of a magnetic conductor carrying an ac electrical current immersed in dc external magnetic field, has attracted a lot of attention [1]. The MI effect offers an appropriate scenario of research in: (i) basic science, (ii) characterization of soft magnetic materials, and (iii) technological applications such as magnetic sensors and devices [1].

Generally, the designers and manufacturers of magnetic sensors seek high sensitive MI materials. Instead, low field sensitivity but step-like flatten response makes this effect a genius candidate for specific application such as magnetic tags and labels [2]. This MI response can be achieved by post-production techniques in the presence of a heat source such as furnace annealing, current Joule heating and laser irradiations [2-7]. These techniques can realign the magnetic domains and their orientations and consequently influence the magnetic anisotropy and the MI effect. Various kinds of magnetic structures can be realized by suitable post-production treatments [2-7]. A local processing by laser irradiation, which can influ-

* corresponding author; e-mail: m-mohseni@cc.sbu.ac.ir ence only a part of an amorphous material, is used as a heat source to anneal amorphous ferromagnetic materials $[2,3]$.

In laser annealing technique, two important points have to be taken into account: (i) distribution across the laser beam is not homogeneous, (ii) non-annealed regions have an extremely different temperature from the laser irradiated region. Therefore, a spatial nonuniform annealed region is created. As can be seen in Fig. 1a, sample is hot at the center of the annealed region and cools down far from the center. The nonuniform heat distribution in a large area can create an inhomogeneous magnetic structure. Interestingly, laser annealing can influence the direction of magnetization along its radiation, normal to the sample surface [5]. In further details, the magnetization response of annealed region against magnetic field is not similar to that of non-annealed regions. On the other hand, magnetization of the annealed region has been bound by a pinning energy distributed uniformly in the annealed region. Interestingly, sharp peak which is normally observed in the field dependent MI effect disappears and flattened and wide field dependent MI response is detectable. Subsequently, transverse magnetic permeability as well as the MI effect of the whole sample is reduced and magnetization curves show a Barkhausen-like noise caused by the changes of irregular magnetic domains located in the junction between annealed and non-annealed regions [2]. Consequently, neglecting the second term of anisotropy in the MI expression can be a major source of error.

In this paper, we present a theoretical model of low frequency MI effect and magnetization process of lo- 


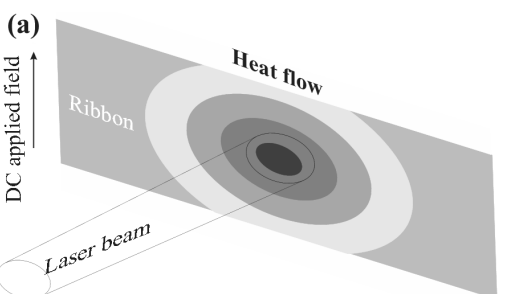

(b) $\quad \mathrm{d}+\mathrm{x} \quad \mathrm{d}-\mathrm{x}$

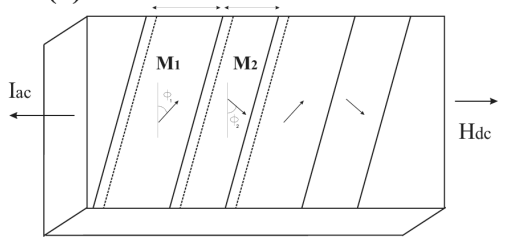

Fig. 1. (a) Schematic diagram of laser irradiation; (b) schematic representation of magnetic domains of a ribbon.

cal annealing of amorphous magnetic ribbons by laser light. Here, to calculate the MI effect, first order uniaxial anisotropy for non-annealed region in the plane of sample [8], Fig. 1b, and second order anisotropy normal to the sample surface in the annealed regions are taken into account. We found out that the MI effect of sample, as a multi-anisotropy structure, includes annealed and non-annealed regions, can be theoretically demonstrated by averaging different magnetic domains owner various anisotropies. The model can explain the experimental results, qualitatively.

\section{Theoretical formalism}

The behavior of impedance, $Z$, with field and frequency is determined by the factor $\sqrt{\mu \omega}[1]$; hence, it is dictated by the response of the domains to the longitudinal field $H$ and the ac current $I$. The frequency and field dependence of the transverse permeability control the MI response of the sample. To start the theoretical objects, we recall the relations used in Ref. [8] to obtain the magnetic susceptibility. To calculate the susceptibility, we used a two-domains model which is schematically presented in Fig. 1b. Domains stated at angles $\phi_{1}, \phi_{2}$, and their wall displacement is represented by $x$. We write the free energy terms for the system as

$$
\begin{aligned}
& U_{\mathrm{A}}=K_{1}\left[\alpha \sin ^{2} \phi_{1}-(1-\alpha) \sin ^{2} \phi_{2}\right] \\
& +K_{2}\left[\alpha \sin ^{4} \phi_{1}-(1-\alpha) \sin ^{4} \phi_{2}\right], \\
& U_{z t}=M_{\mathrm{s}} h\left[(1-\alpha) \cos ^{2} \phi_{2}-\alpha \cos ^{2} \phi_{1}\right], \\
& U_{z}=M_{\mathrm{s}} H\left[(\alpha-1) \sin ^{2} \phi_{2}-\alpha \sin ^{2} \phi_{1}\right], \\
& U_{\mathrm{W}}=\frac{1}{2} k A x^{2},
\end{aligned}
$$

in which the $U_{\mathrm{A}}$ is the anisotropy energy, $U_{z t}$ and $U_{z}$ are the Zeeman (ac and dc parts) and wall energy which can be written as $U_{\mathrm{W}} \cdot K_{1}$ and $K_{2}$ are the first and second order of anisotropy constant, $\alpha=1 / 2+x / d$ is the fraction of the volume occupied by the domains at angle $\phi, d$ is the domain width, $x$ is the wall displacement, $M_{\mathrm{s}}$ is saturation of magnetization, $A$ is the wall area per unit volume and $k$ is the domain wall stiffness. $H$ is external dc applied magnetic field and $h$ is the field produced by ac current, $I$, in transverse direction. To obtain the transverse susceptibility which physically behaves as MI effect, we have to find the angles $\phi_{1}, \phi_{2}$ and $x$ by $\partial U / \partial \phi_{1}=\partial U / \partial \phi_{2}=\partial U / \partial x=0$. By applying the aforementioned conditions and solving equations we can obtain those variables. The transverse susceptibility can be given by

$$
\begin{aligned}
\chi_{\mathrm{t}} & =\frac{M_{\mathrm{t}}}{h}=\frac{M_{\mathrm{s}}}{h} \\
& \times\left[\frac{1}{2}\left(\cos \phi_{1}-\cos \phi_{2}\right)+\frac{x}{d} \cos \phi_{1}+\cos \phi_{2}\right] .
\end{aligned}
$$

The equations have been solved numerically and physical results have been selected for the purposed study.

\section{Results and discussion}

Effect of second order anisotropy is brought out in calculations by using the parameters $K=K_{1} / K_{2}$, where $K$ varies between 0 and 1 . Figure 2 represents the calculated square transverse susceptibility which is convenient to MI for different values of $K$. When $K=0$, the giant MI (GMI) effect is for materials without considering $K_{2}$. An increase in $K$ decreases the MI response and increases the necessary field to achieve the MI peak. It can be due to decrease in the share of initial magnetic domains in the transverse direction by existence of pinning centers at annealed regions [3-5]. To understand the MI response of the whole sample which consists of a complicated magnetic domain structure, we consider some regions with different magnetic anisotropy constant and average over their magnetic susceptibility with various values of $K$. Figure 3 shows the experimental (top) and theoretical (bottom) results of MI response of laser irradiated Co-based amorphous alloys. The experimental curves in Fig. 3 are for laser irradiated samples at $60 \mathrm{~s}$ (a), $90 \mathrm{~s}$ (c) and $120 \mathrm{~s}$ (e). One third middle area of each sample has been irradiated by laser with power of $1 \mathrm{~W}$ [4]. More details about the experiments were published in Ref. [3]. We can observe that the peak behaviors of MI measured in experiments is qualitatively described by the theory. We average the magnetic susceptibility with values of $K=0,0.05,0.1,0.2,0.3,0.4$, 0.5 for Fig. $3 \mathrm{~b}$; and add $K=0.6,0.7$ to the values used in Fig. 3b for Fig. 3d. For Fig. 3f, all values of $K$ in Fig. 3d are used again, but a double weight for $K=0.5$, $0.6,0.7$ is considered and $K=0.4$ is omitted. In spite of some irregularity observed in the data used in theoretical processes, we can generally deduce that increasing the annealing time will increase the value of $K$. 


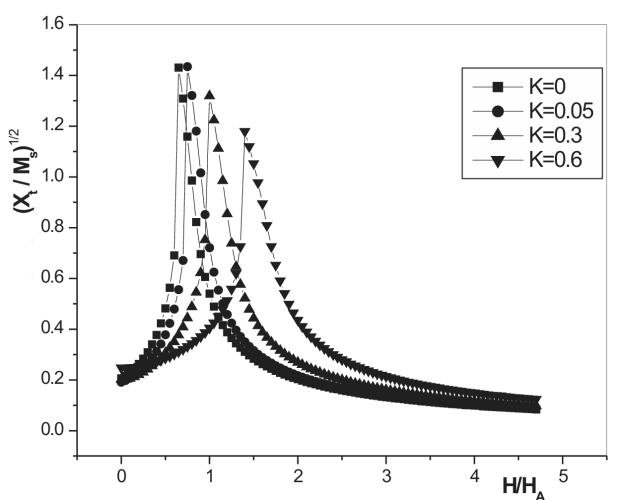

Fig. 2. Field dependence of calculated MI for various values of anisotropy constants.
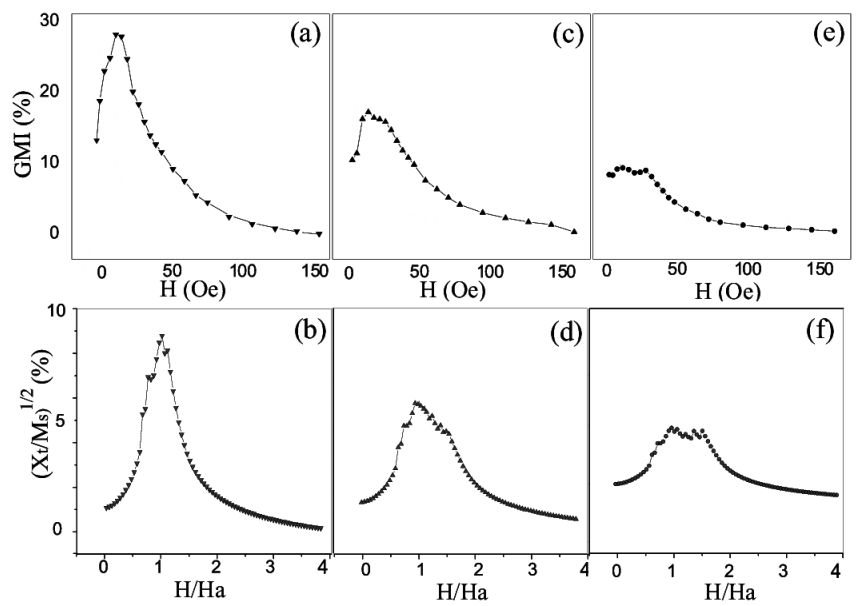

Fig. 3. Field dependence of MI at $250 \mathrm{kHz}$ for laser irradiated Co-based amorphous ribbons (top) and calculated MI curves (bottom) at various irradiation time $60 \mathrm{~s}(\mathrm{a}, \mathrm{b}), 90 \mathrm{~s}(\mathrm{c}, \mathrm{d})$, and $120 \mathrm{~s}(\mathrm{e}, \mathrm{f})$.

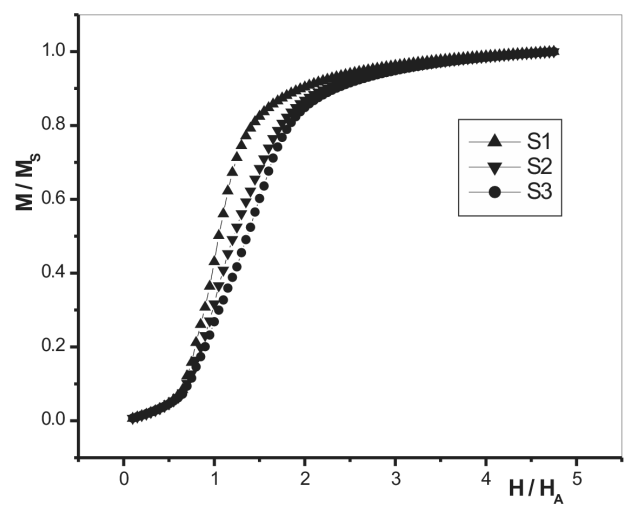

Fig. 4. Calculated initial transverse magnetization curves for those samples represented in Fig. 3.
We expect that in annealed case, the initial magnetization curve behaves much slower than when the domains stated sharply at transverse direction, or $K=0$. To investigate this effect we can roughly integrate the magnetic susceptibility over its all values against applied magnetic field to achieve the initial magnetization curve. Figure 4 shows the initial magnetization curve for all samples studied in Fig. 3. As it is observed, samples have irradiated more, or have large $K$, saturate later. It is due to existence of pinning centers or heterogeneity near the annealed region which makes an incoherent rotation of magnetization against the applied magnetic field.

\section{Conclusion}

We have presented a comprehensive theoretical approach to low frequency MI effect, which accounts for the higher anisotropy constants. Magnetic inhomogeneities produced by laser irradiation of magnetic amorphous ribbons have been investigated in detail. Inhomogeneous magnetic anisotropy which is induced during the irradiation makes a wide peak in MI effect of the samples and increases the magnetic field to reach the MI peaks. Slow saturation of magnetization is predicted by this model. The theoretical model can apply further for heterogeneous magnetic materials for developing new MI based sensors and devices.

\section{References}

[1] M. Knobel, M. Vazquez, L. Kraus, in: Handbook of Magnetic Materials, Ed. K.H.J. Buschow, Vol. 15, Elsevier, Amsterdam 2003, p. 497.

[2] A.N. Grigorenko, L.V. Panina, S.I. Sandacci, D.P. Makhnovskiy, D.J. Mapps, I.Y. Khrushchev, M.V. Dubov, V. Larin, A. Torkunov, Appl. Phys. Lett. 81, 4790 (2002).

[3] S.E. Roozmeh, M.M. Tehranchi, M. Ghanaatshoar, S.M. Mohseni, M. Parhizkari, H. Ghomi, H. Latifi, J. Magn. Magn. Mater. 304, e633 (2005).

[4] M.M. Tehranchi, M. Ghanaatshoar, S.M. Mohseni, M. Moradi, J. Non-Cryst. Solids 353, 896 (2007).

[5] M. Sorescu, J. Alloy. Comp. 280, 251 (1998).

[6] P. Tiberto, F. Vinai, O. Rampado, H. Chiriac, T.A. Ovari, J. Magn. Magn. Mater. 196-197, 388 (1999).

[7] H. Chiriac, F. Vinai, T.A. Ovari, C.S. Malinesen, F. Barariu, P. Tiberto, Mater. Sci. Eng. A 226-228, 646 (1997).

[8] F.L.A. Machado, S.M. Rezende, J. Appl. Phys. 79, 6558 (1996). 